\title{
HST WFPCII Observations of the Inner HR Car Nebula
}

\author{
Stephen Hulbert ${ }^{1}$, Antonella Nota ${ }^{1}$, Mark Clampin ${ }^{1}$, Claus Leitherer ${ }^{1}$, \\ Anna Pasquali ${ }^{2}$, Norbert Langer ${ }^{3}$, and Regina Schulte-Ladbeck ${ }^{4}$ \\ 1 Space Telescope Science Institute \\ 2 ST-ECF/European Southern Observatory \\ 3 Institut für Theoretische Physik und Astrophysik, Potsdam \\ 4 University of Pittsburgh
}

\begin{abstract}
We present $\mathrm{H} \alpha$ observations of the inner region of the HR Carinae nebula. The inner nebula looks clumpy and material appears to be present only to the east of the central star. A jet-like filament stretches away from the central star to the southeast.
\end{abstract}

HR Car, one of the few known galactic Luminous Blue Variables (LBVs), was originally reported to be surrounded by an "arc-shaped" nebula with a jet-like feature by Hutsemékers \& Van Drom (1991). Later imaging revealed a bipolar morphology. Filamentary structure is evident over the $19^{\prime \prime} \times 37^{\prime \prime}$ extent of the bipolar lobes with the SE lobe most prominent (Weis et al. 1997, Nota et al. 1997). Spectroscopic observations of the SE lobe show blue-shifted radial velocities up to $100 \mathrm{~km} \mathrm{~s}^{-1}$ while the $\mathrm{NW}$ lobe shows red-shifted radial velocities of the same magnitude. The bipolar nebula is oriented at $125-135^{\circ} \mathrm{E}$ of $\mathrm{N}$ in the plane of the sky-it is tilted between $40^{\circ}$ and $60^{\circ}$ into the plane of the sky. The innermost part of the nebula was first identified by Clampin et al. (1995) as the "waist" of the bipolar nebula-similar to the corresponding feature found in $\eta$ Car. Figure 1 (left) shows the nebula as seen from the ground with coronography (Clampin et al. 1995). Figure 2 (left) shows the model of the bipolar nebula including the equatorial waist (adapted from Nota et al. 1997).

$\mathrm{H} \alpha$ images of HR Car were obtained during 1996 using the HST instrument configuration of $\mathrm{WFPC} 2+\mathrm{F} 656 \mathrm{~N}$. Observations were separated in time such that the nominal roll of HST had changed by $22^{\circ}$. Multiple exposures at each epoch were combined to remove cosmic rays. The complex background caused by the bright central source was first modeled and then subtracted. Field stars were used to rotate and align the images from the two epochs. By combining two images with different orientations we were able to remove the HST secondary spider shadows from the images and were forced to mask out only the central region of the image where the CCD was saturated.

$¿$ From the most recent HST observations of HR Car, the inner nebula appears clumpy. Figure 1 (right) shows the innermost $17^{\prime \prime}$ of the HR Car nebula as seen by WFPC2-the central star is centered in the circular masked region. We have measured four of the largest clumps of material in the waist; 
the clumps have masses of: A-0.07, B-0.02, C-0.01, D-0.04 $M_{\odot}$. The four clumps account for about half of the total mass, $0.40 \mathrm{M} \odot$, of the inner nebula based on integrated $H \alpha$ measurements. Figure 2 (right) shows the individual clumps. The apparent "clump" marked with an asterisk is actually a previously identified star (Hutsemékers \& Van Drom 1991).

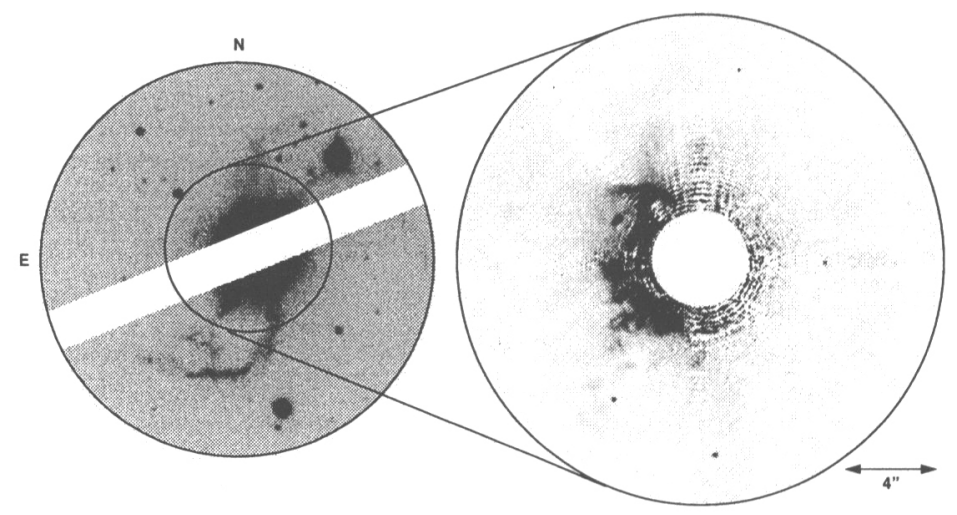

Fig. 1. Inner HR Car nebula: (left) ground-based image of HR Car bipolar nebula. (right) HST WFPCII image of HR Car inner nebula.

The inner nebula is arranged asymmetrically around the central starmaterial is visible only to the east of the central star. The visible material appears roughly elliptical in shape shape with a size of $3^{\prime \prime} \times 6^{\prime \prime}$ and an orientation roughly $\mathrm{E}$ of $\mathrm{N}$. The asymmetric distribution of material near the central star is curious but not inconsistent with it being the waist of the bipolar nebula. If the visible portion represents only part of the waist, an elliptical fit to the structure of the complete waist agrees with the equatorial plane being tilted into the plane of the sky about $58^{\circ}$-consistent with the range of tilts for the lobes of the bipolar nebula (see figure 2). In addition, the orientation of the elliptical fit on the sky $\left(30^{\circ}\right)$ is in gross agreement with the polarization results of Clampin et al. (1995).

The expansion time scale of the inner nebula is comparable to that of bipolar nebula. The nebular expansion velocity is $100 \mathrm{~km} \mathrm{~s}^{-1}$ yielding a dynamical time scale of $5 \times 10^{3}$ years for the bipolar nebula (Nota et al. 1997). The dynamical time scale for the clumps in the inner nebula (about $3^{\prime \prime}$ away from the central star) is about $1.6 \times 10^{3}$ using an average velocity of $50 \mathrm{~km} \mathrm{~s}^{-1}$ taken from previous spectroscopic observations of the nebula (Nota 
et al. 1997). The agreement of these two time scales is, in turn, consistent with the identification of the inner nebula as the waist of the bipolar nebula.

A jet-like filament (JLF) is evident emanating from the direction of the central object. The filament extends to at least $8^{\prime \prime}$ from the central star and has an orientation of $140^{\circ} \mathrm{E}$ of $\mathrm{N}$-both the JLF and the bipolar nebula are arrayed in the same direction in the plane of the sky. This filament points directly at the rather unusual "fork-shaped" filament associated with the SE lobe of the bipolar nebula-apparently bisecting the tines of the fork. Previous spectroscopic measures that coincide with the location of the JLF show high velocity blue-shifted components along the filament of $50-100 \mathrm{~km} \mathrm{~s}^{-1}$ up to $10 "$ away from central object (Nota et al. 1997). Figure 2 shows the location of the JLF in the sky as well as in the model of the nebula.

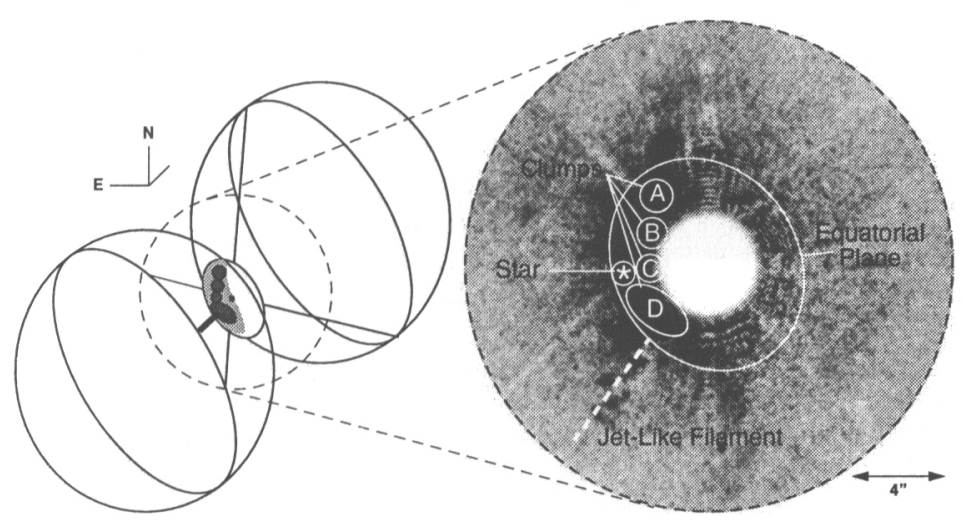

Fig. 2. HR Car Nebula Model: (left) Schematic of bipolar nebula. (right) Identification of major clumps and jet-like filament in HR-CAR waist.

\section{References}

Clampin, M., Schulte-Ladbeck, R.E., Nota, A., Robberto, M., Paresce, F., Clayton, G.C. (1995): AJ 110, 251

Hutsemékers, D., Van Drom, E. (1991): A\&Ap 248, 141

Nota, A., Livio, M., Clampin, M.(1995): ApJ 448, 788

Nota, A., Smith, L., Pasquali, A., Clampin, M., Stroud, M. (1997): ApJ 486, 338

Weis, K., Duschl, W.J., Bomans, D.J., Chu, Y.-H., Joner, M.D. (1997): A\&Ap 320, 568 


\section{Discussion}

N. Trams: The TIMMI images of HR Car (Voors et al. 1997) I showed in my talk yesterday show not only the small inner nebula, but also evidence for emission from the other side of the central star, which would be coming from the backside of the "waist" of the bipolar nebula.

R. Waters: The HST images nicely confirm the presence of the asymmetric inner nebula, which was discovered by Voors et al (1997). The IR images show the innermost part of the nebula $\left(<2^{\prime \prime}\right)$ and clearly demonstrate that the orientation of the inner nebula is similar to that of the outer nebula.

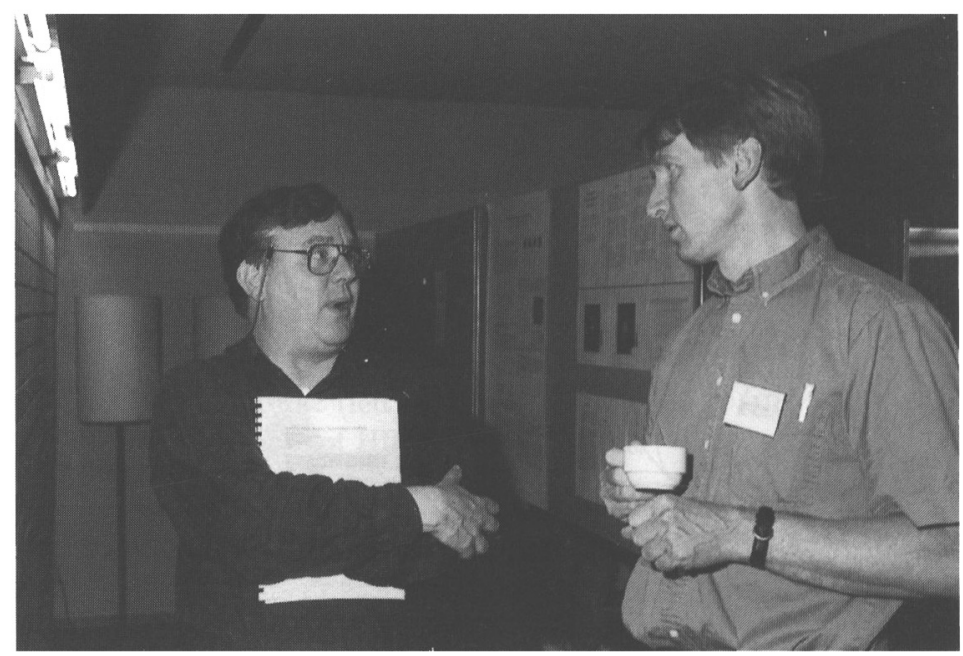

Peredur Williams and Stephen White 